\title{
In situ atomistic mechanisms of detwinning in nanocrystalline AuAg alloy
}

\author{
Libo Fu', Chengpeng Yang ${ }^{1}$, Yan $\mathrm{Lu}^{1}$, Jiao Teng ${ }^{2}$, Deli Kong ${ }^{1}$, Yizhong Guo ${ }^{1}$, Ze Zhang ${ }^{3}$, \\ Lihua Wang ${ }^{1 *}$ and Xiaodong Han $^{1 *}$
}

\begin{abstract}
Detwinning is an important plastic deformation mechanism that can significantly affect the mechanical properties of twin-structured metals. Although many detwinning mechanisms have been proposed for pure metals, it is unclear whether such a deformation model is valid for nanocrystalline alloys because of the lack of direct evidence. Here, the atomicscale detwinning deformation process of a nanocrystalline AuAg alloy with an average grain size of $\sim 15 \mathrm{~nm}$ was investigated in situ. The results show that there are three types of detwinning mechanisms in nanocrystalline AuAg alloys. The first type of detwinning results from grain boundary migration. The second type of detwinning occurs through combined layer-by-layer thinning and incoherent twin boundary migration. The last one occurs through incoherent twin boundary migration, which results from the collective motion of partial dislocations in an array.
\end{abstract}

Keywords: twin-structured, detwinning, in situ, transmission electron microscopy, atomic scale

\section{INTRODUCTION}

Face-centered cubic (FCC)-structured metals with grown nanotwins have attracted great interest because of their ultrahigh strength and excellent ductility compared to their twin-free counterparts [1-4]. Because the mechanical properties of twinstructured metals are directly related to their deformation mechanisms, a large number of studies have been conducted to study the deformation mechanism of twin-structured metals in the last decades [1-8]. It is well established that the strengthening of twin-structured metals results from dislocations intersecting with coherent twin boundaries (CTBs), which was confirmed by both molecular dynamics (MD) simulations and experimental investigations [1,5,6,9-12]. In contrast, for the softening mechanism, in situ atomic-scale observations of the detwinning process have rarely been studied, especially for twinstructured nanocrystalline (NC) metals with grain sizes of $\sim 15 \mathrm{~nm}[3,9,10,13-16]$. In addition to dislocation-CTB intersection, several MD simulations and experiments have indicated that detwinning can also significantly affect the mechanical properties of twin-structured metals $[7,10,11,17]$. The atomicscale mechanism of detwinning is crucial, which may provide clues for designing twin-structured metals with high strength and excellent ductility $[1,10,16-19]$.
Previous MD studies have proposed that the detwinning process results from partial dislocation emission from CTBgrain boundary (GB) intersection and glide along the plane adjacent to the CTB [12-15]. This process leads to CTB migration, which in turn leads to twin thinning in a layer-bylayer manner [16,18,20-23]. Other studies have suggested that detwinning can also result from incoherent TB (ITB) migration $[8,19,24,25]$. For twins in FCC-structured metals, an ITB consists of an array of Shockley partial dislocations on each $\{111\}$ plane [19,24-27]. The detwinning process results from the partial dislocation array moving back to the boundary or free surface $[13,16,19,25]$. In contrast, previous experiments have indicated that this ITB migration can originate from partial dislocations moving successively at the ITB, which results in steps on the TBs $[14,15,19,28,29]$. However, previous modeling has also predicted that ITB migration can occur via the collective glide of partial dislocations in an array from the ITB [19,24,2931]. However, most previous studies are based on the results of coarse-grained or ultrafine-grained metals, and whether other detwinning mechanisms exist for NC metals with grain sizes of $\sim 15 \mathrm{~nm}$ remains unclear. More importantly, the proposed detwinning mechanism is based on experiments on pure metals, and its validity for alloy metals has not been confirmed. Thus, it will be interesting to provide in situ atomic-scale evidence of the deformation mechanism of detwinning in NC metallic alloys.

In this study, the deformation behavior of an NC AuAg alloy with an average grain size of $\sim 15 \mathrm{~nm}$ was investigated in situ at the atomic scale using a custom-built device $[32,33]$. The results show that there are three types of detwinning mechanisms: GB migration-induced detwinning, combined layer-by-layer thinning and ITB migration, and ITB migration. We provide direct in situ atomic-scale evidence that full dislocations can hinder ITB migration, which has only been predicted in modeling thus far.

\section{EXPERIMENTAL SECTION}

An NC AuAg alloy thin film with a uniform composition (Figs S1 and S2) was deposited on a single-crystal $\mathrm{NaCl}$ substrate using a co-sputtering strategy with an ATC-1800F direct-current magnetron sputtering system. Au and Ag targets (>99.99\% purity) were used. Before sputtering, the air pressure of the chamber was $\sim 2 \times 10^{-7}$ Torr $(1$ Torr $=133.3 \mathrm{~Pa})$. During sputtering, high-purity Ar gas (99.995\% purity) was fed into the chamber, and the chamber pressure was maintained at $\sim 2 \times$

\footnotetext{
${ }^{1}$ Beijing Key Lab of Microstructure and Properties of Advanced Materials, Beijing University of Technology, Beijing 100124, China

${ }^{2}$ Department of Material Physics and Chemistry, University of Science and Technology Beijing, Beijing 100083, China

${ }^{3}$ Department of Materials Science, Zhejiang University, Hangzhou 310027, China

* Corresponding authors (emails: wlh@bjut.edu.cn (Wang L); xdhan@bjut.edu.cn (Han X))
} 
$10^{-3}$ Torr. The sputtering powers of $\mathrm{Au}$ and Ag were $\sim 19$ and $\sim 23 \mathrm{~W}$, respectively, corresponding to a nominal deposition rate of $\sim 8.5 \mathrm{~nm} \mathrm{~min}{ }^{-1}$. The AuAg alloy film with a thickness of $\sim 25 \mathrm{~nm}$ was deposited on the $\mathrm{NaCl}$ substrate and then annealed at $200^{\circ} \mathrm{C}$ for $1 \mathrm{~h}$. Transmission electron microscopy (TEM) was used to reveal the boundaries and interiors of the grains (Fig. S2). Then, the grain size was determined from the TEM images by measuring the length of lines drawn between GBs as shown in Fig. S2. Subsequently, the statistical grain size distribution was obtained by measuring more than 300 grains from different regions of the NC thin film.

Under an optical microscope, the thin film on the $\mathrm{NaCl}$ substrate was attached to a bimetallic extensor using epoxy resin. Subsequently, the $\mathrm{NaCl}$ substrate was etched with water, and the AuAg thin film became free-standing and remained on the bimetallic extensor. The thin film was thinned further to a thickness of $\sim 20 \mathrm{~nm}$ using a Fischione 1040 NanoMill. Next, the bimetallic extensor was loaded in a heated holder with a doubletilt capability and placed in an FEI Titan G2 TEM $(300 \mathrm{kV})$. The bimetallic extensor was composed of two thin layers of materials with a large mismatch between their coefficients of thermal expansion, which could produce large displacements at relatively low operating temperatures $\left(<50^{\circ} \mathrm{C}\right)$. During testing, the bimetal extender was controlled to exert a tensile force on the sample by accurately raising the temperature using a temperature controller, which could achieve slow and gentle deformation with a strain rate below $\sim 4 \times 10^{-3} \mathrm{~s}^{-1}$.

\section{RESULTS AND DISCUSSION}

Fig. 1 shows a series of high-resolution TEM (HRTEM) images, which show that GB migration resulted in detwinning during the deformation of the NC AuAg alloy thin film. To track the detwinning mechanism more clearly, $G_{i-j}$ is defined as the GB between grains $\mathrm{G}_{i}$ and $\mathrm{G}_{j}$, and the $\mathrm{GB}$ is marked by a white dotted line. As can be seen, the plastic deformation of a nanotwin grain $\left(\mathrm{G}_{1}\right)$ is governed by GB migration, leading to a decrease in the twinned area. Fig. la shows a typical HRTEM image captured at the early stage of tensile loading, and it can be seen that both $G_{1}$ and $G_{3}$ exhibit the [110] axis lattice of FCC AuAg. As indicated in Fig. 1a, a nanotwin $\left(\mathrm{T}_{1-2}\right)$ with a thickness of nine atomic layers crosses $G_{1}$ and intersects the $G B$ s of $G_{1-2}$ and $\mathrm{G}_{1-3}$; the TBs are indicated by yellow solid lines. With increasing the strain, as shown in Fig. $1 \mathrm{~b}, \mathrm{G}_{1-3}$ moves toward the left side, decreasing the lengths of $\mathrm{T}_{1}$ and $\mathrm{T}_{2}$ from 4.5 to $2.6 \mathrm{~nm}$ and from 10.2 to $8.4 \mathrm{~nm}$, respectively. With continuous strain- ing, as shown in Fig. $1 c, G_{1-3}$ moves toward the left side, and $G_{1-2}$ moves toward the right side, decreasing the lengths of $\mathrm{T}_{1}$ and $\mathrm{T}_{2}$ to $\sim 2.4$ and $5.6 \mathrm{~nm}$, respectively. With further straining, the migration of $G_{1-2}$ and $G_{1-3}$ leads to the annihilation of $T_{1}$ and $T_{2}$. In addition, there was no noticeable change in the misorientation angle between $G_{1}$ and $G_{3}$ during the $G B$ migration process, indicating detwinning was mainly governed by GB migration. This GB migration allowed the detwinning process to occur without the need for partial dislocation nucleation from the CTB-GB intersection or the movement of partial dislocations at the ITB. Thus, this type of detwinning process is important in NC metals because GB migration is more easily triggered in small-sized grains $[7,12]$. During the deformation process, the lattices in $G_{1}$ and $G_{3}$ exhibited no obvious changes, indicating that there was no obvious out-of-plane rotation or global tilt of the specimen during deformation.

Fig. 2 displays several typical magnified HRTEM images along the [110] axis that show the combined layer-by-layer thinning and ITB migration. This detwinning process occurred in two steps: partial dislocation nucleation from the CTB-GB intersection, followed by ITB migration. Fig. 2a shows the image obtained at the initial time $(0 \mathrm{~s})$, where the loading direction is labeled by the double white arrow. Fig. $2 \mathrm{~b}-\mathrm{g}$ provide six magnified HRTEM images that correspond to the yellow framed region in Fig. 2a, and the times are 0, 6.5, 15.5, 37, 43.5, and $51 \mathrm{~s}$. In Fig. $2 b$, there is a full dislocation $\mathrm{D}_{1}$ interacting with the CTB. By employing Burgers circuits around the dislocation cores in the magnified HRTEM image in Fig. S3, it is found that there is an extra half-plane on $\{111\}$ planes. This configuration represents a typical full dislocation with a Burgers vector of $\boldsymbol{b}=\frac{1}{2}<011>$, which was frequently observed in previous studies [33-35]. With increasing strain, as shown in Fig. 2c, two steps on the CTB can be found at different positions, and step 1 is an obstacle created by full dislocation $\mathrm{D}_{1}$. As can be seen, on the left side of step 1, there are three atomic layers associated with the twin, and on the right side of step 1, the twin has two atomic layers. This feature should be associated with a partial dislocation emitted from the right side of the GB-TB intersection and move in the left direction during straining. The glide of the partial dislocation diminished an additional twinned layer behind its movement, resulting in one-atomic-layer thinning. For step 2, the structural feature was nearly the same as step 1, indicating that its origin was also a partial dislocation emission and glide from the right side of the GB-TB intersection. With continuous straining, step 1 remained because it was obstructed
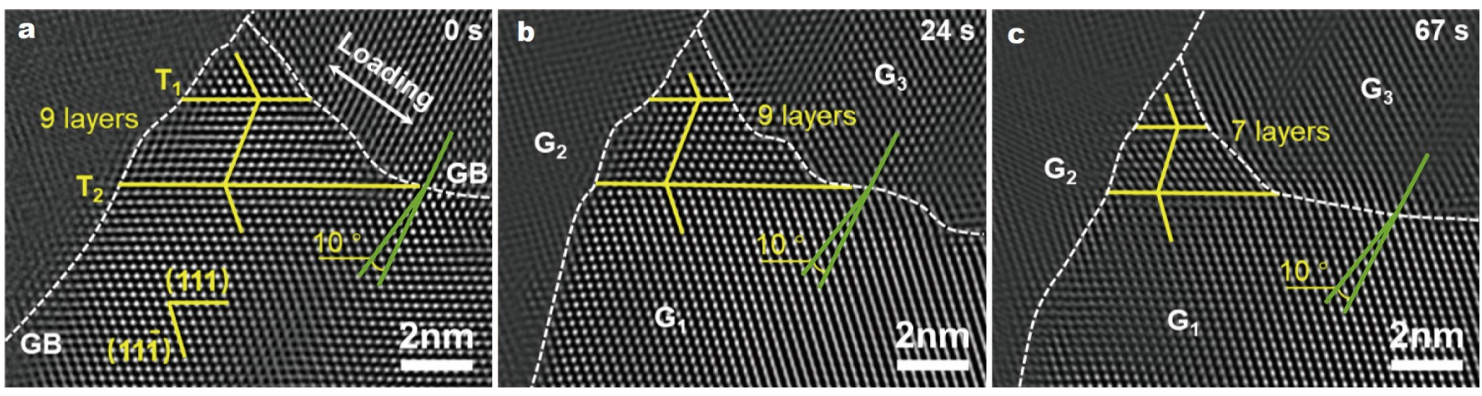

Figure 1 In situ atomic-scale observation of the detwinning deformation dominated by the GB migration in nanograins of AuAg alloys. The GB is denoted by the white dotted line. The CTBs are denoted by the yellow solid line. The white double arrow indicates the loading direction. The misorientation angle between $G_{1}$ and $G_{3}$ is denoted by the green solid line. (a-c) With the GB migration, the nanotwin suffered the plastic deformation, and both the length and the thickness were reduced. 

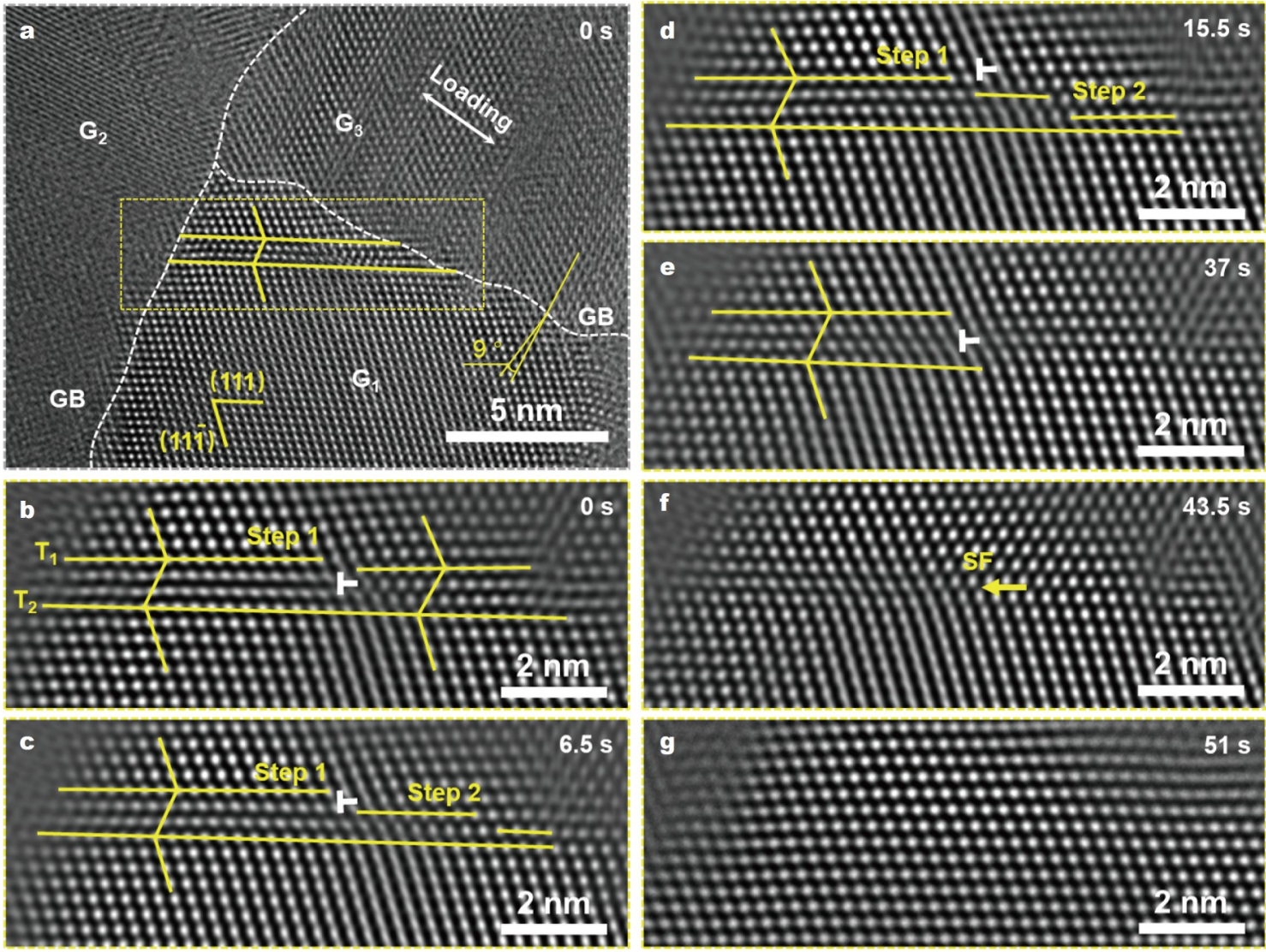

Figure 2 In situ atomic-scale observations of detwinning dominated by the combined layer-by-layer thinning and ITB migration in nanograins of AuAg alloys. (a) HRTEM image showing the morphology of nanotwins. The misorientation angle between $G_{1}$ and $G_{3}$ is $9^{\circ}$. The scale bar is $5 \mathrm{~nm}$. (b-g) Series of magnified HRTEM images showing the detwinning process through layer-by-layer thinning in AuAg alloy specimens. The scale bars are $2 \mathrm{~nm}$.

by full dislocation $D_{1}$, whereas step 2 moved in the left direction (Fig. 2d). As the strain was increased, a third partial dislocation emission from the right GB-TB intersection and glide toward the left side occurred. The movement of these three dislocations was prevented by $D_{1}$. Thus, we can observe an ITB consisting of three partial dislocations confined in $G_{1}$. This is direct evidence that a full dislocation can hinder ITB migration and delay the detwinning process, which was only predicted in previous classical modeling $[10,25,36]$. With further straining, two partial dislocations moved toward $\mathrm{G}_{1-2}$ and were annihilated. As a result, a stacking fault (SF) was observed in $\mathrm{G}_{1}$. With extensive straining, the third partial dislocation moved toward $\mathrm{G}_{1-2}$ and was annihilated again, leading to the defect-free grain. Thus, our in situ atomic-scale results show that when a full dislocation creates an obstacle, the detwinning process is more likely to undergo two stages: combined layer-by-layer thinning and ITB migration. In the first stage, partial dislocation nucleation occurs because of the GB-TB intersection, and then an obstacle is created by the full dislocation, resulting in an ITB confined in the grain. In the second stage, the detwinning process results from successive movement of partial dislocations at the ITB toward the GB, which ultimately leads to the grown twin annihilation.

Fig. 3 shows the in situ atomic-scale observation of the third type of detwinning process in the NC AuAg alloy specimen, where detwinning occurs through ITB migration that results from the collective motion of partial dislocations at the ITB. In
Fig. 3a, one can observe a nanotwin with a thickness of seven atomic layers and a length of $6.8 \mathrm{~nm}$. The left side of the nanotwin intersects with the GB, and the right side is embedded in the nanograin featuring an ITB that consists of an array of partial dislocations on each $\{111\}$ plane. In order to track the detwinning process, the position of the CTB is indicated by yellow lines, and the ITB is indicated by a dashed yellow line. During loading, the nanotwin suffered plastic deformation, as shown in Fig. 3b, and the thickness changed from seven layers to six layers, resulting from the movement of partial dislocations on the ITB toward the left side of the GB. With further straining, detwinning continued through ITB migration. As shown in Fig. 3b-d, the ITB moved toward the left side, decreasing the length of the nanotwin from 6.8 to $4.8 \mathrm{~nm}$. With further loading, the ITB continued to move to the left, decreasing the length of the nanotwin to $\sim 3.0 \mathrm{~nm}$ (Fig. 3e). Further straining led to twin annihilation, as shown in Fig. 3f. During straining, the ITB was nearly straight without an obvious irregular shape or a step on the $\mathrm{CTB}$, indicating the detwinning process was governed by the collective motion of partial dislocations in an array on the ITB $[19,24]$.

To estimate the detwinning rate in the alloy specimens, Fig. 4 shows the length of the nanotwins as a function of time. The black square, red circle, and blue triangle represent detwinning via combined layer-by-layer thinning and ITB migration, ITB migration, and GB migration, respectively. As can be seen, the rate of detwinning resulting from GB migration is the slowest. 

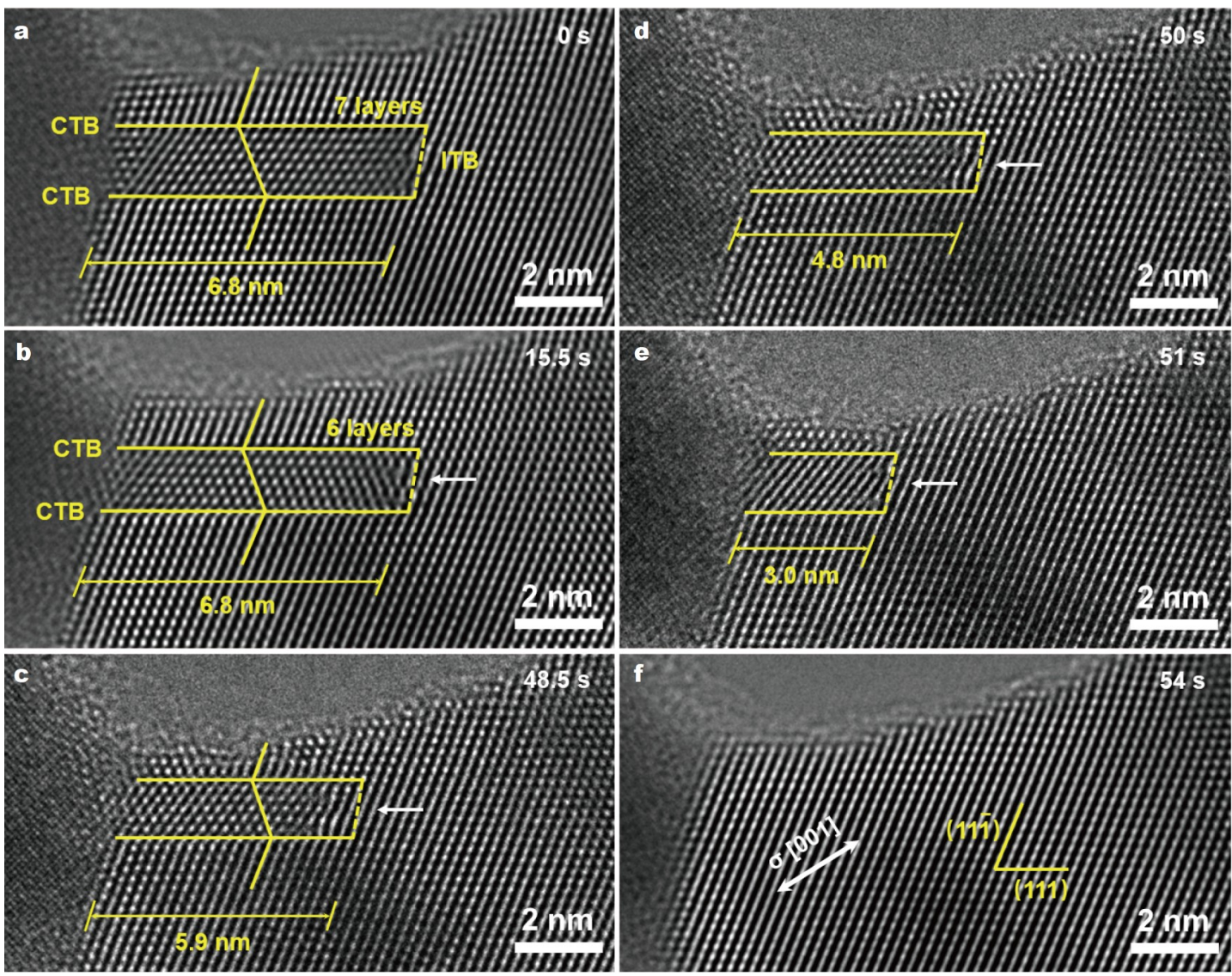

Figure 3 In situ atomic-scale observation of the ITB migration-induced detwinning process in the twin lamellae. (a, b) Nanotwin with seven atomic layers in thickness underwent thinning through straining, and the thickness decreased to six atomic layers. (c-e) During straining, the ITB migrated in the left direction, decreasing the length of the nanotwin from 5.9 to $3.0 \mathrm{~nm}$. (f) With the extensive loading, the ITB moved to the left continuously until it contacted the GB, and the nanotwin disappeared. The detwinning deformation completed, and the crystal returned to the ideal lattice at this moment. The white double arrow in the picture indicates the loading direction, along the crystal direction of the [001]. The scale bars are $2 \mathrm{~nm}$.

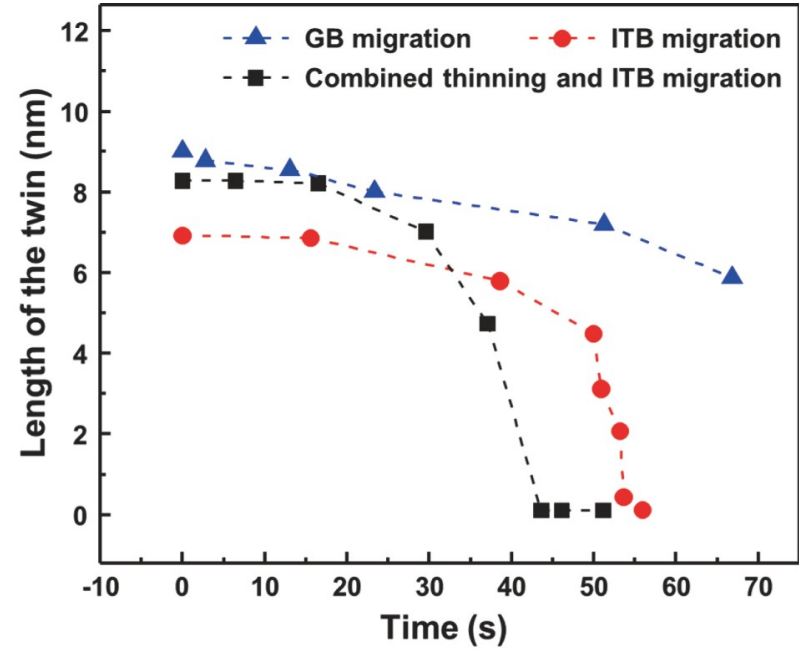

Figure 4 Length of the nanotwins as a function of time after the detwinning process occurred in three different deformation modes. Three different symbols represent the mechanisms of detwinning, and dashed lines of different colors represent the fitting curves of the corresponding experimental data.

This is because the detwinning rate is controlled by the GB migration, which should be relatively slow at room temperature.
For the combined layer-by-layer thinning and ITB migration, the rate falls into two regions corresponding to the two stages of detwinning. In the first stage, the detwinning rate is relatively slow because the full dislocation on the CTB hinders the partial dislocation motion. In the second stage, the ITB is generated, and the detwinning rate becomes very fast. This is also consistent with the rate of detwinning resulting from ITB migration (red circle) being faster than the other two detwinning mechanisms. These results indicate that the dislocation-CTB interaction can delay the detwinning process and contribute to strain hardening, whereas ITB migration can easily occur, which can supply large plasticity $[1,7,10,11]$.

According to previous studies, the plastic deformation will switch from dislocation behavior to GB plasticity as the grain size is below $\sim 15 \mathrm{~nm}$ [34]. This strong grain size effect on deformation can significantly affect the observed detwinning mechanisms. In the current study, the grain size of the thin film was in the range of $\sim 3-38 \mathrm{~nm}$ (with an average grain size of $\sim 15 \mathrm{~nm}$ ). For twin-structured grains with sizes above $\sim 15 \mathrm{~nm}$, intragrain dislocation activities are more likely to occur $[37,38]$, in which the driving force for dislocation motion is smaller than that for GB plasticity $[39,40]$. Thus, the detwinning resulting from the collective motion of partial dislocations (Fig. 3) or combined layer-by-layer thinning and ITB migration (Fig. 2) is more likely to occur in relatively large-sized grains. In addition, 
the interactions of partial dislocations or full dislocations with TBs may also affect the detwinning process. In small-sized grains, the driving force for GB plasticity is smaller than that for dislocation activity $[35,41,42]$; thus, GB migration-induced detwinning is more likely to occur (Fig. 1). Although both GB migration and ITB migration that resulted in detwinning are driven by applied stress, their mechanisms are very different. GB migration-induced detwinning is governed by stress-driven GB atom diffusion and GB atom shuffling coupled to GB migration [43]. The ITB consists of an array of partial dislocations [19,24], and ITB migration-induced detwinning involves the motion of these partial dislocation under an applied stress $[13,19,44]$. In addition to the grain size, the loading mode can also affect the detwinning mechanism $[16,30,45,46]$. For example, previous MD simulations indicated that under torsion, the detwinning in twin-structured metallic nanowires results from the rotation of the $\{111\}$ plane [45]. This rotation enables all the atomic layers above twin plane "C" (CABCBAC) to be restored to the perfect FCC structure (CABCABC) after being rigidly twisted at an angle of $60^{\circ}$. Under the conventional deformation model, detwinning resulting from dislocation nucleation and slip or $\mathrm{TB}$ migration was also observed in the TiAl alloy [46], which is similar to the results shown in Figs 2 and 3 in the current study.

In the current study, in situ atomic-scale observations reveal that there are three types of detwinning mechanisms. The first type is the result of GB migration, which occurs more easily in very small nanograins. The second type is more likely to occur in relatively large nanograins, in which dislocations are highly active and thus have a high possibility of interacting with the CTB $[7,10,16,47,48]$. Once a dislocation is pinned by the CTB, it can hinder ITB migration and delay the detwinning process, leading to strain hardening, which has only been predicted in classical modeling thus far [12,37,49-51]. The third type of detwinning occurs via the collective glide of partial dislocations, which results in ITB migration; this is consistent with the detwinning mechanism previously reported for pure metals $[13,19,24,48]$. The first two types of detwinning have rarely been reported in binary solid-solution NC alloys. They are also different from previous studies on pure metals, in which detwinning results from partial dislocation glide on the plane adjacent to the CTB in a layer-by-layer manner or from partial dislocation motion on the ITB $[11,19,52,53]$. It should be noted that the solution atoms, secondary phases, precipitated phases, and local chemical composition segregation in alloys can also affect the dislocation activity $[46,51,54,55]$, which affects the detwinning mechanism. Particularly for those alloys containing more than three types of solution atoms, such as medium-entropy or highentropy alloys, the detwinning mechanism should be different from those observed in the current study and in pure metals. As shown in previous studies, there are noticeable fluctuations in the elemental distribution in medium- or high-entropy alloys [51,54,56-58]. This local chemical ordering can cause the nucleation energy and dislocation motion pathways to vary between regions [59,60], which would affect the detwinning model. Thus, we believe that the detwinning mechanisms in medium- or high-entropy alloys will exhibit new behaviors, which require further study.

\section{CONCLUSIONS}

In summary, using our custom-built device, the atomic-scale and time-resolved detwinning process in NC alloys was observed in situ. We discovered three types of detwinning mechanisms: GB migration, combined layer-by-layer thinning and ITB migration, and collective motion of partial dislocations in an array, resulting in ITB migration. We showed that dislocationCTB interaction could delay detwinning and contribute to strain hardening, whereas ITB migration could supply large plasticity. Our experimental results provide deeper insight into the fundamental detwinning mechanism in metallic alloys and also provide important clues for achieving high strength and high plasticity of twin-structured alloy NC metals.

\section{Received 28 June 2021; accepted 17 August 2021;}

published online 24 September 2021

1 Jang D, Li X, Gao H, et al. Deformation mechanisms in nanotwinned metal nanopillars. Nat Nanotech, 2012, 7: 594-601

2 Pan Q, Lu L. Dislocation characterization in fatigued $\mathrm{Cu}$ with nanoscale twins. Sci China Mater, 2015, 58: 915-920

3 Zhu Y. Catch twin nucleation in action at atomic scale. Sci China Mater, 2018, 61: 1019-1020

4 Cheng $\mathrm{G}$, Zhang $\mathrm{Y}$, Chang $\mathrm{TH}$, et al. In situ nano-thermomechanical experiment reveals brittle to ductile transition in silicon nanowires. Nano Lett, 2019, 19: 5327-5334

5 Wang J, Zeng Z, Weinberger CR, et al. In situ atomic-scale observation of twinning-dominated deformation in nanoscale body-centred cubic tungsten. Nat Mater, 2015, 14: 594-600

6 Pan Q, Zhou H, Lu Q, et al. History-independent cyclic response of nanotwinned metals. Nature, 2017, 551: 214-217

7 Wang L, Du K, Yang C, et al. In situ atomic-scale observation of grain size and twin thickness effect limit in twin-structural nanocrystalline platinum. Nat Commun, 2020, 11: 1167

8 Lu Y, Shu X, Liao X. Size effect for achieving high mechanical performance body-centered cubic metals and alloys. Sci China Mater, 2018, 61: 1495-1516

9 Wang YM, Sansoz F, LaGrange T, et al. Defective twin boundaries in nanotwinned metals. Nat Mater, 2013, 12: 697-702

10 Lu N, Du K, Lu L, et al. Transition of dislocation nucleation induced by local stress concentration in nanotwinned copper. Nat Commun, 2015, 6: 7648

11 Chen Y, An X, Zhou Z, et al. Size-dependent deformation behavior of dual-phase, nanostructured CrCoNi medium-entropy alloy. Sci China Mater, 2021, 64: 209-222

12 Li X, Lu L, Li J, et al. Mechanical properties and deformation mechanisms of gradient nanostructured metals and alloys. Nat Rev Mater, 2020, 5: 706-723

13 Lee S, Im J, Yoo Y, et al. Reversible cyclic deformation mechanism of gold nanowires by twinning-detwinning transition evidenced from in situ TEM. Nat Commun, 2014, 5: 3033

14 Li BQ, Sui ML, Mao SX. Pseudoelastic stacking fault and deformation twinning in nanocrystalline Ni. Appl Phys Lett, 2010, 97: 241912

15 Zhang X, Misra A. Superior thermal stability of coherent twin boundaries in nanotwinned metals. Scripta Mater, 2012, 66: 860-865

16 An X, Ni S, Song M, et al. Deformation twinning and detwinning in face-centered cubic metallic materials. Adv Eng Mater, 2020, 22: 1900479

17 Lu K, Lu L, Suresh S. Strengthening materials by engineering coherent internal boundaries at the nanoscale. Science, 2009, 324: 349-352

18 Cao Y, Wang YB, An XH, et al. Concurrent microstructural evolution of ferrite and austenite in a duplex stainless steel processed by highpressure torsion. Acta Mater, 2014, 63: 16-29

19 Wang L, Teng J, Kong D, et al. In situ atomistic deformation mechanisms of twin-structured nanocrystal Pt. Scripta Mater, 2018, 147: 103-107

20 Kumar KS, Suresh S, Chisholm MF, et al. Deformation of electrodeposited nanocrystalline nickel. Acta Mater, 2003, 51: 387-405

21 Van Swygenhoven H, Derlet PM, Frøseth AG. Stacking fault energies and slip in nanocrystalline metals. Nat Mater, 2004, 3: 399-403

22 Shute CJ, Myers BD, Liao Y, et al. High-pressure torsion of copper 
samples containing columns of highly aligned nanotwins. Scripta Mater, 2011, 65: 899-902

23 Guo YZ, Sun T, Fu LB, et al. In situ atomic-scale observation of dislocation behaviors in twin-structured $\mathrm{Pt}$ nanocrystals. Sci China Technol Sci, 2021, 64: 599-604

24 Wang J, Li N, Anderoglu O, et al. Detwinning mechanisms for growth twins in face-centered cubic metals. Acta Mater, 2010, 58: 2262-2270

25 An XH, Song M, Huang Y, et al. Twinning via the motion of incoherent twin boundaries nucleated at grain boundaries in a nanocrystalline $\mathrm{Cu}$ alloy. Scripta Mater, 2014, 72-73: 35-38

26 Li BQ, Li B, Wang YB, et al. Twinning mechanism via synchronized activation of partial dislocations in face-centered-cubic materials. Scripta Mater, 2011, 64: 852-855

27 Lin TC, Liang CL, Wang SB, et al. Inhibiting the detrimental $\mathrm{Cu}$ protrusion in $\mathrm{Cu}$ through-silicon-via by highly (111)-oriented nanotwinned Cu. Scripta Mater, 2021, 197: 113782

28 Hong CS, Tao NR, Huang X, et al. Nucleation and thickening of shear bands in nano-scale twin/matrix lamellae of a $\mathrm{Cu}-\mathrm{Al}$ alloy processed by dynamic plastic deformation. Acta Mater, 2010, 58: 3103-3116

29 Li N, Wang J, Huang JY, et al. Influence of slip transmission on the migration of incoherent twin boundaries in epitaxial nanotwinned $\mathrm{Cu}$. Scripta Mater, 2011, 64: 149-152

30 Liang Y, Yang X, Gong M, et al. Slip transmission for dislocations across incoherent twin boundary. Scripta Mater, 2019, 166: 39-43

31 Zhang Y, Guo J, Ming W, et al. Atomic-scale study on incoherent twin boundary evolution in nanograined Cu. Scripta Mater, 2020, 186: 278281

32 Wang L, Teng J, Wu Y, et al. Size dependence of dislocation activities and independence on theoretical elastic strain limit in Pt nanocrystals revealed by atomic-resolution in situ investigation. Mater Today Nano, 2018, 2: 1-6

33 Fu L, Yang C, Wei R, et al. In situ atomic-scale observation of $\mathrm{AuCu}$ alloy nanowire with superplasticity and high strength at room temperature. Mater Today Nano, 2021, 15: 100123

34 Meyers MA, Mishra A, Benson DJ. Mechanical properties of nanocrystalline materials. Prog Mater Sci, 2006, 51: 427-556

35 Cao Y, Ni S, Liao X, et al. Structural evolutions of metallic materials processed by severe plastic deformation. Mater Sci Eng-R-Rep, 2018, 133: $1-59$

36 Li X, Wei Y, Lu L, et al. Dislocation nucleation governed softening and maximum strength in nano-twinned metals. Nature, 2010, 464: 877880

37 Ni S, Wang YB, Liao XZ, et al. Effect of grain size on the competition between twinning and detwinning in nanocrystalline metals. Phys Rev B, 2011, 84: 235401

38 Ovid'ko IA, Valiev RZ, Zhu YT. Review on superior strength and enhanced ductility of metallic nanomaterials. Prog Mater Sci, 2018, 94: 462-540

39 Yaghoobi M, Voyiadjis GZ. Size effects in fcc crystals during the high rate compression test. Acta Mater, 2016, 121: 190-201

40 Cao P. The strongest size in gradient nanograined metals. Nano Lett, 2020, 20: 1440-1446

41 Wang L, Teng J, Liu P, et al. Grain rotation mediated by grain boundary dislocations in nanocrystalline platinum. Nat Commun, 2014, 5: 4402

42 El-Awady JA. Unravelling the physics of size-dependent dislocationmediated plasticity. Nat Commun, 2015, 6: 5926

43 Han J, Thomas SL, Srolovitz DJ. Grain-boundary kinetics: A unified approach. Prog Mater Sci, 2018, 98: 386-476

44 Wei Y. The kinetics and energetics of dislocation mediated de-twinning in nano-twinned face-centered cubic metals. Mater Sci Eng-A, 2011, 528: $1558-1566$

45 Zhou H, Li X, Wang Y, et al. Torsional detwinning domino in nanotwinned one-dimensional nanostructures. Nano Lett, 2015, 15: 60826087

46 Zeng Y, Li X. Atomistic simulations of high-temperature creep in nanotwinned TiAl alloys. Extreme Mech Lett, 2021, 44: 101253

47 Li N, Wang J, Misra A, et al. Twinning dislocation multiplication at a coherent twin boundary. Acta Mater, 2011, 59: 5989-5996
$48 \mathrm{Li} \mathrm{BQ}$, Sui ML, Li B, et al. Reversible twinning in pure aluminum. Phys Rev Lett, 2009, 102: 205504

49 Zhou H, Li X, Qu S, et al. A jogged dislocation governed strengthening mechanism in nanotwinned metals. Nano Lett, 2014, 14: 5075-5080

50 You Z, Lu L. Deformation and fracture mechanisms of nanotwinned metals. Natl Sci Rev, 2017, 4: 519-521

51 Chen S, Oh HS, Gludovatz B, et al. Real-time observations of TRIP induced ultrahigh strain hardening in a dual-phase CrMnFeCoNi highentropy alloy. Nat Commun, 2020, 11: 826

52 Wang YD, Liu W, Lu L, et al. Low temperature deformation detwinning: A reverse mode of twinning. Adv Eng Mater, 2010, 12: 906-911

53 Sun L, He X, Lu J. Nanotwinned and hierarchical nanotwinned metals: A review of experimental, computational and theoretical efforts. npj Comput Mater, 2018, 4: 6

54 Yang T, Zhao YL, Tong Y, et al. Multicomponent intermetallic nanoparticles and superb mechanical behaviors of complex alloys. Science, 2018, 362: 933-937

55 Peng S, Wei Y, Gao H. Nanoscale precipitates as sustainable dislocation sources for enhanced ductility and high strength. Proc Natl Acad Sci USA, 2020, 117: 5204-5209

56 He J, Wang Q, Zhang $\mathrm{H}$, et al. Dynamic deformation behavior of a facecentered cubic FeCoNiCrMn high-entropy alloy. Sci Bull, 2018, 63: 362-368

57 Du XH, Li WP, Chang HT, et al. Dual heterogeneous structures lead to ultrahigh strength and uniform ductility in a Co-Cr-Ni medium-entropy alloy. Nat Commun, 2020, 11: 2390

58 Guo L, Gu J, Gong X, et al. CALPHAD aided design of high entropy alloy to achieve high strength via precipitate strengthening. Sci China Mater, 2020, 63: 288-299

59 Li QJ, Sheng H, Ma E. Strengthening in multi-principal element alloys with local-chemical-order roughened dislocation pathways. Nat Commun, 2019, 10: 3563

60 Ding Q, Zhang Y, Chen X, et al. Tuning element distribution, structure and properties by composition in high-entropy alloys. Nature, 2019, 574: $223-227$

Acknowledgements This work was supported by Beijing Natural Science Foundation (Z180014), Beijing Outstanding Young Scientists Projects (BJJWZYJH01201910005018), and the National Natural Science Foundation of China (51771104).

Author contributions $\mathrm{Fu} \mathrm{L}$, Yang $\mathrm{C}$ and $\mathrm{Lu} \mathrm{Y}$ conducted the in situ TEM experiments; Fu L, Kong D and Guo Y conducted the data statistics; Teng J synthesized the AuAg alloy nanocrystals; Han X, Zhang Z and Wang L designed the project and guided the research; Fu L, Han X and Wang L wrote the manuscript. All authors have discussed and approved the results and conclusions.

Conflict of interest The authors declare that they have no conflict of interest.

Supplementary information Supporting data are available in the online version of the paper.

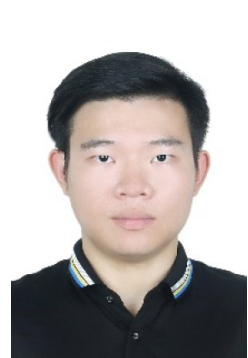

Libo Fu is a PhD candidate at Beijing Key Laboratory and the Institute of Microstructure and Properties of Advanced Materials, Beijing University of Technology. His current research is focused on in-situ straining electron microscopy investigation of the deformation behaviors of nanocrystalline face-centered cubic metallic materials at the atomic scale. 


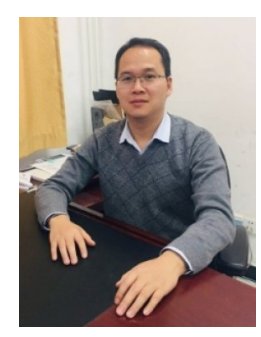

Lihua Wang is currently a professor at Beijing University of Technology. He has been engaged in "insitu experimental research on the mechanical behavior of materials at the atomic scale". For the first time, the atomic-scale in-situ observation of the deformation mechanism of the metal nano-polycrystalline system has been realized. He authored and co-authored more than 60 international journal publications (including Nature Commun, Phys Rev Lett, Acta Mater, etc.) with total citations exceeding 3000 .

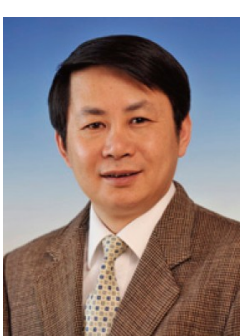

Xiaodong Han is a professor at Beijing University of Technology. He originally developed the atomic-level in-situ dynamic characterization method of the mechanical behavior of materials, and increased the spatial resolution of the in-situ characterization technology of the mechanical behavior of materials from nanometers to picometer scales, achieving an order of magnitude improvement. His research mainly focuses on the physical and chemical properties of materials, the mechanical behavior of materials from micro-nano to atomic resolution in a wide temperature range (room temperature to $1200^{\circ} \mathrm{C}$ ) and environmental conditions.

\section{$\operatorname{AuAg}$ 合金纳米晶去孪晶机制的原位原子尺度研究}

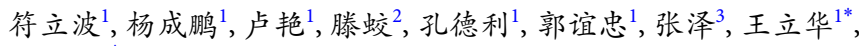
韩晓东 ${ }^{1 *}$

摘要 去孪晶是影响金属力学性能的重要塑性变形机制. 本文对平均 晶粒尺寸为 $15 \mathrm{~nm}$ 的 $\mathrm{AuAg}$ 合金纳米晶进行了原位拉伸试验, 首次在原 子尺度上研究了合金纳米晶的去孪晶机制. 结果表明合金纳米晶中存 在三种去孪晶机制, 第一种是晶界发生相向迁移的结果, 这在小尺寸晶 粒中更容易发生; 第二种是通过孪晶逐层减薄和非共格孪晶界迁移相 结合的方式进行, 在去孪晶过程中, 去孪晶速率会由于位错与共格孪晶 界相互作用的影响而减缓, 这仅在以往的经典建模中预测到; 第三种是 由非共格孪晶界迁移控制, 这是多个偏位错集体滑移的结果. 前两种类 型的去孪晶过程在过去很少被报道，这表明固溶体合金纳米晶中的去 孪晶机制不同于纯金属. 